\title{
Suction Drain Tip Culture after Spine Surgery: Can It Predict a Surgical Site Infection?
}

\author{
Jae-Sung Ahn, Ho-Jin Lee, Eugene Park, Il-Young Park, Jae Won Lee \\ Department of Orthopaedic Surgery, Chungnam National University School of Medicine, Daejeon, Korea
}

\begin{abstract}
Study Design: Retrospective clinical study.
Purpose: To assess the diagnostic value of suction drain tip culture in patients undergoing primary posterior spine surgery.

Overview of Literature: To date, the diagnostic value of suction drain tip culture for predicting surgical site infection (SSI) has not been firmly established in orthopedic or spinal surgery.

Methods: In total, 133 patients who underwent primary posterior spine surgery from January 2013 to April 2015 were included in this retrospective study. Patients diagnosed with infective disease or condition was excluded. The suction drain tip was cut off approximately $5 \mathrm{~cm}$ from its far end. The sample was sent to the microbiological laboratory of the hospital for culture analysis. Any signs of infection, such as wound discharge or dehiscence, fever, chills, or chronic pain, were recorded. The culture outcome, identification of bacteria, and postoperative transition of the serum C-reactive protein level were also recorded in all patients. The wounds were followed up for a minimum of 3 months.

Results: A positive drain tip culture was found in 48 patients (36.1\%), of whom, 6 developed SSI. The sensitivity of drain tip culture for SSI after primary posterior spine surgery was $60.0 \%$, and the specificity was $65.9 \%$. The association between the incidence of positive suction tip culture and SSI was not statistically significant. Among the 48 positive drain tip cultures, there was no significant association between the occurrence of SSI and virulence of isolated bacteria. There was no significant association between drain tip culture positivity and the duration of drainage, or between the rate of SSI and duration of drainage.

Conclusions: Suction drain tip culture analysis is a poor predictor of SSI after primary posterior spine surgery. Routine use of a drain tip culture is not supported by the results of this study.
\end{abstract}

Keywords: Spine surgery; Surgical site infection; Drain tip culture; Diagnostic value; Bacteria

\section{Introduction}

Closed-suction drainage is an effective tool for minimizing hematoma formation around the surgical site and reducing the infection rate [1,2]. Many surgeons consider wound hematoma to be an ideal medium for bacterial colonization and postoperative surgical site infection (SSI)
[1]. Therefore, suction drainage has been used postoperatively by the majority of orthopedic surgeons to reduce the potential for infection $[2,3]$.

Numerous studies on the use of closed-suction drainage after surgery have reported the results of suction drain tip culture after trauma or elective surgeries [3-8], but the relationship between a positive tip culture and SSI remains

Received Jul 29, 2015; Revised Sep 28, 2015; Accepted Oct 1, 2015

Corresponding author: Ho-Jin Lee

Department of Orthopedic Surgery, Chungnam National University School of Medicine,

33 Munwha-ro, Jung-gu, Daejeon 35015, Korea

Tel: +82-42-280-7353, Fax: +82-42-252-7098, E-mail: leeleo98@gmail.com 
controversial [2,3,5,6,8-12]. To date, the diagnostic value of suction drain tip culture for predicting SSI has not been firmly established in orthopedic or spinal surgery.

The relationship between the timing of drain removal and the occurrence of SSI after spine surgery also remains unclear. In several studies, longer durations of suction drain use were associated with higher rates of SSI after surgery $[5,13]$. Therefore, this study assessed the diagnostic value of suction drain tip culture in patients who underwent primary posterior spine surgery. We determined: (1) the sensitivity, specificity, and positive and negative predictive values of suction drain tip culture; (2) the correlation between the bacterial species cultured from the drain tip and the occurrence of SSI; and (3) the association with the duration of drainage, drain tip culture positivity, and the development of SSI.

\section{Materials and Methods}

In total, 133 patients who underwent elective posterior spine surgery from January 2013 to April 2015 were included in this retrospective study. Operations in our study were performed by one spine surgeon at our institute. The patients comprised 79 men and 54 women with an average age of 64.2 years (range, 32-89 years). The patients were classified according to American Society Anesthesiologists (ASA) status to collect individual preoperative medical conditions. Operations were most frequently performed on the lumbar spine (55.6\%), followed by the cervical spine (35.3\%). Fusion was the leading type of operation followed by laminoplasty, and degeneration was the most common pathology for surgery (Table 1). The serum C-reactive protein level (s-CRP) was routinely checked before surgery in all patients with primary spinal pathology, and patients were included if the s-CRP was in the normal range. Patients diagnosed with an infective disease or conditions were excluded, as well as those with revision or anterior spine surgery.

All patients underwent surgery under the same aseptic conditions. The skin was sterilized with a $10 \%$ aqueous povidone iodine solution, and the surgical field was covered with a sterile drape. The suction drain was inserted in all patients during the surgery, and the perforated ends of the drain tubes were placed bilaterally in the proximal portion of the midline longitudinal incision. A prophylactic antibiotic was injected intravenously for 5 days $(1.0 \mathrm{~g}$ of cefmetazole; 1 hour before skin incision and every 12
Table 1. Demographic data

\begin{tabular}{|c|c|}
\hline Characteristic & Patients $(n=133)$ \\
\hline Age (yr) & $64.23 \pm 11.74$ \\
\hline \multicolumn{2}{|l|}{ Sex } \\
\hline Male & $79(59.4)$ \\
\hline Female & $54(40.6)$ \\
\hline \multicolumn{2}{|l|}{ ASA status } \\
\hline I & $9(6.8)$ \\
\hline$\|$ & $88(66.2)$ \\
\hline III & $32(24.0)$ \\
\hline IV & $4(3.0)$ \\
\hline V & 0 \\
\hline $\mathrm{VI}$ & 0 \\
\hline \multicolumn{2}{|c|}{ Location of operation } \\
\hline C-spine & $47(35.3)$ \\
\hline T-spine & $12(9.0)$ \\
\hline L-spine & $74(55.6)$ \\
\hline \multicolumn{2}{|l|}{ Type of operation } \\
\hline Laminoplasty & $37(27.8)$ \\
\hline Decompression & $16(12.0)$ \\
\hline Fusion & $80(60.2)$ \\
\hline \multicolumn{2}{|l|}{ Pathology } \\
\hline Trauma & $11(8.3)$ \\
\hline Degeneration & $119(89.5)$ \\
\hline Neoplasm & $3(2.2)$ \\
\hline
\end{tabular}

Values are presented as mean \pm standard deviation or number (\%). ASA, American Society Anesthesiologists.

hours postoperatively).

The suction drain was removed when the amount of fluid that drained from the operative site was $<50 \mathrm{~mL}$ in patients who underwent cervical and thoracic surgery and $<100 \mathrm{~mL}$ in those who underwent lumbar surgery. We disinfected the skin surrounding the drain using a $10 \%$ aqueous povidone iodine solution before drain removal [1]. The suction drain tip was cut off approximately $5 \mathrm{~cm}$ from its far end using single-use sterile scissors, and the sample was sent to the microbiological laboratory of the hospital for cultural analysis.

SSIs were defined according to the criteria of Centers for Disease Control and Prevention [14]. A superficial SSI was determined when an infection involved only the skin and the subcutaneous tissue of the incision, in contrast to a deep SSI involving the deep soft-tissue muscle and fascia.

Any signs of infection, such as wound discharge or 
dehiscence, fever, chills, or chronic pain, were recorded. The culture outcome, identification of bacteria, and postoperative transition of the s-CRP were also recorded in all patients. Wounds were followed up for a minimum of 1 year.

Statistical analysis was performed using the SPSS ver. 12.0 (SPSS Inc., Chicago, IL, USA). Fisher's exact test and the chi-square test were used for categorical variables. For continuous variables, an independent $t$-test was used for normally distributed data, and the Mann-Whitney $\mathrm{U}$ test was used for non-normally distributed data. A $p$-value of $<0.05$ was taken to indicate statistical significance.

\section{Results}

Table 2 shows the relationship between drain tip culture and SSI. A positive drain tip culture was found in 48 patients (36.1\%), 6 of whom developed SSI. Ten SSIs occurred, four of which yielded negative tip cultures. All SSIs underwent management including wound opening, abscess culture, debridement of infected tissue, massive irrigation, and abscess removal. Two out of 10 SSIs were superficial SSI and 8 were deep. All the superficial SSI cases showed negative tip culture results. For all the patients with SSI, none underwent revision spine surgery. All but six patients with a positive tip culture required

Table 2. The relation between drain tip culture and SSI

\begin{tabular}{lcc} 
Suction tip culture & SSIs $(\mathrm{n})$ & No SSIs $(\mathrm{n})$ \\
\hline Positive & 6 & 42 \\
Negative & 4 & 81 \\
\hline Total & 10 & 123 \\
\hline
\end{tabular}

SSI, surgical site infection. no additional therapy because there were no other signs of wound infection. In total, 81 of 85 negative tip culture cultures were negative for SSI (Table 2). From our analysis, the sensitivity of drain tip culture for SSI after primary posterior spine surgery was $60.0 \%$, and the specificity was $65.9 \%$. The positive value of drain tip culture for SSI was $12.5 \%$, and the negative predictive value was $95.3 \%$. The association between the incidences of a positive drain tip culture and the development of SSI was not statistically significant $(p=0.195)$ (Table 2).

Table 3 lists the bacterial species that were cultured from patients with SSI and the drain tips. Six of 48 positive drain tip culture wounds developed SSIs; in each of these cases, the culture from the wound abscess and that from the drain tip showed growth of the same bacterial species with similar antibiotic sensitivity. The total number of SSIs was 10. In eight cases, we identified the bacterial species in the wound abscess culture; six of these were positive drain tip culture SSIs and two were negative drain tip culture SSIs. Four coagulase-negative staphylococci (CNS) infections and two Staphylococcus aureus infections were identified among the six positive drain tip culture SSIs. In the 48 positive drain tip culture cases, there was no significant association between the occurrence of SSI and the virulence of the isolated bacteria $(p=0.439)$. Bacterial species were identified in two of the four negative drain tip culture SSIs: one was a CNS species and the other was Enterococcus faecalis.

Table 4 shows the associations among the duration of drainage, drain tip culture positivity, and occurrence of SSI. We removed the drain after surgery after an average of 4.51 days (range, 2-16 days). There was no significant association between drain tip culture positivity and the duration of drainage ( $p=0.738$ ), nor between the rate of

Table 3. Bacterial species cultured from SSI cases and drain tip

\begin{tabular}{lcc} 
Bacterial species & SSIs (positive tip cultured+negative tip cultured) & Drain tip cultures \\
Coagulase-negative staphylococci & $5(4+1)$ & 39 \\
Staphylococcus aureus & $2(2+0)$ & 5 \\
$\begin{array}{l}\text { Staphylococcus aureus } \\
\text { +Coagulase-negative staphylococci }\end{array}$ & - & 1 \\
Bacillus & - & 1 \\
$\quad$ Enterococcus faecalis & $1(0+1)$ & 1 \\
$\quad$ Enterobacter cloacae & - & 1 \\
Total & $8(6+2)$ & 48 \\
\hline
\end{tabular}

SSI, surgical site infection. 
Table 4. The association between duration of drainage, positive tip culture and SSI

\begin{tabular}{lccc} 
Drainage time (hr) & Case & Positive culture & SSI \\
\hline $24-48$ & 4 & 1 & 0 \\
$48-72$ & 28 & 14 & 4 \\
\hline $72-96$ & 47 & 12 & 1 \\
$96-120$ & 28 & 12 & 3 \\
$120-144$ & 16 & 4 & 0 \\
$>144$ & 10 & 48 & 10 \\
\hline Total & 133 & & 2 \\
\hline
\end{tabular}

SSI, surgical site infection.

SSI and the duration of drainage $(p=0.954)$.

\section{Discussion}

We performed this study on spine surgery patients to assess the diagnostic value of culture analysis of suction drain tips, which are commonly used by orthopedic surgeons. The spine surgeries were limited to primary posterior operations. Patients with revision surgeries, infectious conditions, and other infections were excluded. The primary aims of our study were to determine the diagnostic value of suction drain tip culture for predicting SSI and elucidate the correlation between the bacterial species cultured from the drain tip and the occurrence of SSI. We also analyzed the association between the duration of drainage, drain tip culture positivity, and development of SSI.

In our study, the association between the incidences of a positive drain tip culture and the development of SSI was not significant (Table 2). Drain tip culture had no predictive value for future SSI. According to the literature, no spine surgery studies have evaluated the diagnostic or prognostic value of drain tip culture analysis for SSI. Furthermore, no definite conclusions can be drawn from the results of orthopedic surgery, including joint arthroplasty. Several authors have reported the absence of an association between drain tip culture positivity and the development of SSI [5,6,9-12]. Petsatodis et al. [10] prospectively cultured 110 suction drain tips after total hip arthroplasty (THA) (73 primary and 37 noninfected revisions); 8 cultures were positive, but no SSIs were recorded. Takada et al. [12] reported that 1,380 closed-suction drain tips were cultured after primary THA. SSI was observed in four cases $(0.3 \%)$, all of which had a negative drain tip culture.
The sensitivity of drain tip culture for SSI after THA was $0.0 \%$, and the specificity was $99.7 \%$. The positive predictive value was $0.0 \%$, and the negative predictive value was $99.2 \%$. In a study by Weinrauch [11], 393 drain tip cultures were performed in 387 patients (145 hip replacements, 242 knee replacements). Three patients had a positive drain tip culture, none of whom were diagnosed with superficial or deep infection. The sensitivity was $0.0 \%$ and the specificity was $99.2 \%$. All three of these studies concluded that the prognostic value of drain tip culture for determining joint infection was very limited and that its routine use was not supported by the clinical data. However, other authors have reported a significant correlation between a positive drain tip culture and SSI in orthopedic surgeries [2,3,8]. Sorensen and Sorensen [3] reported on 489 clean orthopedic operations: 56 involved a positive drain tip culture, and 5 of these 56 developed an SSI. Sankar et al. [8] described 214 patients who underwent clean orthopedic operations. Six of the 12 positive tip culture wounds developed deep infection. Suction tip cultures had a sensitivity of $75 \%$, a specificity of $97 \%$, and positive and negative predictive values of $50 \%$ and $99 \%$, respectively, for detecting a wound infection. In all of the above studies, however, the strength of the association between the culture results and infection was relatively low.

In other reports, the most frequently described cultured bacteria were $S$. aureus and CNS $[2,3,8,15,16]$. In our study, most of the isolated microorganisms were nonvirulent CNS or bacilli. In total, 39 (81.3\%) of 48 cases involved CNS bacteria. Moreover, even in patients with SSI, nonvirulent bacteria were more prevalent than virulent bacteria (four cases of CNS vs. two cases of S. aureus). We were unable to precisely determine the source of drain tip contamination, but the most plausible hypothesis is that 
during the operation, microorganisms located outside of the body migrated into the patient's deep soft tissue [17]. In the 48 cases of drain tip culture positivity, there was no significant association between the occurrence of SSI and the virulence of the isolated bacteria (Table 3).

No definitive conclusions have been drawn regarding the associations among the drainage duration, drain tip culture positivity, and the development of SSI in orthopedic surgery $[2,3,6,8,12,13]$. Moreover, we found no previous spine surgery studies on this topic. In a study by Drinkwater and Neil [13], 92 drain tip cultures were investigated after clean orthopedic surgery; if drain removal was performed after 24 hours, the drain tip culture positivity rate increased. Takada et al. [12] reported no correlation between the timing of drain removal and the onset of SSI, while a positive drain tip culture was significantly more likely when the drain was removed more than 48 hours postoperatively (after THA in 1,380 closed-suction drain tips). However, Sankar et al. [8] noted no significant correlation between wound infection and the duration of drainage. In a clinical study of 81 THA cases, Overgaard et al. [6] found no correlation between positive drain tip cultures and the duration of drainage. In our study, there was no significant association between drain tip culture positivity and the duration of drainage, nor between the rate of SSI and the duration of drainage (Table 4).

Our study had several limitations. First, we used a retrospective review design that could have resulted in a lack of power in design. For further validation, a prospective trial is necessary. Second, the sample size used in this study was relatively small. Despite these limitations, the study had several strengths. First, to the best of our knowledge, this is the first study on the value of drain tip culture after spine surgery. Numerous studies have evaluated drain tip culture after surgery in general orthopedic surgery, especially after hip or knee arthroplasty. However, we found no such studies involving spine surgery in the literature. Because maintaining a suction drain tip is a common procedure after spine surgery to prevent postoperative neurological injury and SSI due to epidural hematoma $[18,19]$, this study has value as the first investigation of the diagnostic and prognostic value of suction drain tip culture in patients undergoing spine surgery. Although the findings of this article were similar to previous reports for THA or total knee arthroplasty (TKA), our study is worth being reported, because the need and the frequency for suction drain in spine surgery is higher than in THA or TKA. Second, our study was limited to patients undergoing primary posterior spine surgeries without infection. Patients undergoing anterior or revision spine surgeries were excluded to minimize bias.

\section{Conclusions}

Suction drain tip culture analysis is a poor predictor of SSI after primary posterior spine surgery. Routine use of drain tip culture is not supported by the results of this study.

\section{Conflict of Interest}

No potential conflict of interest relevant to this article was reported.

\section{References}

1. Waugh TR, Stinchfield FE. Suction drainage of orthopaedic wounds. J Bone Joint Surg Am 1961;43:93946.

2. Willett KM, Simmons CD, Bentley G. The effect of suction drains after total hip replacement. J Bone Joint Surg Br 1988;70:607-10.

3. Sorensen AI, Sorensen TS. Bacterial growth on suction drain tips: prospective study of 489 clean orthopedic operations. Acta Orthop Scand 1991;62:451-4.

4. Bernard L, Pron B, Vuagnat A, et al. The value of suction drainage fluid culture during aseptic and septic orthopedic surgery: a prospective study of $901 \mathrm{pa}-$ tients. Clin Infect Dis 2002;34:46-9.

5. Girvent R, Marti D, Munoz JM. The clinical significance of suction drainage cultures. Acta Orthop Belg 1994;60:290-2.

6. Overgaard S, Thomsen NO, Kulinski B, Mossing NB. Closed suction drainage after hip arthroplasty: prospective study of bacterial contamination in 81 cases. Acta Orthop Scand 1993;64:417-20.

7. Reilly TJ, Gradisar IA Jr, Pakan W, Reilly M. The use of postoperative suction drainage in total knee arthroplasty. Clin Orthop Relat Res 1986;(208):238-42.

8. Sankar B, Ray P, Rai J. Suction drain tip culture in orthopaedic surgery: a prospective study of 214 clean operations. Int Orthop 2004;28:311-4.

9. Willemen D, Paul J, White SH, Crook DW. Closed suction drainage following knee arthroplasty: effec- 
tiveness and risks. Clin Orthop Relat Res 1991;(264): 232-4.

10. Petsatodis G, Parziali M, Christodoulou AG, Hatzokos I, Chalidis BE. Prognostic value of suction drain tip culture in determining joint infection in primary and non-infected revision total hip arthroplasty: a prospective comparative study and review of the literature. Arch Orthop Trauma Surg 2009;129:1645-9.

11. Weinrauch P. Diagnostic value of routine drain tip culture in primary joint arthroplasty. ANZ J Surg 2005;75:887-8.

12. Takada R, Jinno T, Koga D, Hirao M, Muneta T, Okawa A. Is drain tip culture prognostic of surgical site infection? Results of 1380 drain tip cultures in total hip arthroplasty. J Arthroplasty 2015;30:1407-9.

13. Drinkwater CJ, Neil MJ. Optimal timing of wound drain removal following total joint arthroplasty. J Arthroplasty 1995;10:185-9.

14. Horan TC, Gaynes RP, Martone WJ, Jarvis WR, Emori TG. CDC definitions of nosocomial surgical site infections, 1992: a modification of CDC definitions of surgical wound infections. Infect Control Hosp Epidemiol 1992;13:606-8.
15. Lindgren U, Elmros T, Holm SE. Bacteria in hip surgery: a study of routine aerobic and anaerobic cultivation from skin and closed suction wound drains. Acta Orthop Scand 1976;47:320-3.

16. Zamora-Navas P, Collado-Torres F, de la Torre-Solis F. Closed suction drainage after knee arthroplasty: a prospective study of the effectiveness of the operation and of bacterial contamination. Acta Orthop Belg 1999;65:44-7.

17. Burnett JW, Gustilo RB, Williams DN, Kind AC. Prophylactic antibiotics in hip fractures: a double-blind, prospective study. J Bone Joint Surg Am 1980;62:45762.

18. Lawton MT, Porter RW, Heiserman JE, Jacobowitz R, Sonntag VK, Dickman CA. Surgical management of spinal epidural hematoma: relationship between surgical timing and neurological outcome. J Neurosurg 1995;83:1-7.

19. Morse K, Weight M, Molinari R. Extensive postoperative epidural hematoma after full anticoagulation: case report and review of the literature. J Spinal Cord Med 2007;30:282-7. 